\title{
Noncovalent Approach toward the Construction of Nanofluidic Diodes with pH-Reversible Rectifying Properties: Insights from Theory and Experiment
}

Gonzalo Pérez-Mitta, ${ }^{*}{ }^{\dagger}$ Alberto Albesa, ${ }^{\dagger}$ Facundo M. Gilles, ${ }^{\dagger}$ María Eugenia Toimil-Molares, ${ }^{\dagger}$ Christina Trautmann, ${ }^{\ddagger}$, and Omar Azzaroni* ${ }^{* \dagger}+0$

\author{
${ }^{\dagger}$ Instituto de Investigaciones Fisicoquímicas Teóricas y Aplicadas (INIFTA), Departamento de Química, Facultad de Ciencias \\ Exactas, Universidad Nacional de La Plata, CONICET-CC 16 Suc. 4, La Plata 1900, Argentina \\ ${ }^{\ddagger}$ GSI Helmholtz Centre for Heavy Ion Research GmbH, 64289 Darmstadt, Germany

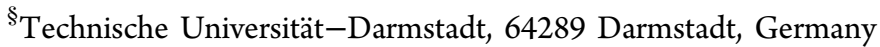

Supporting Information

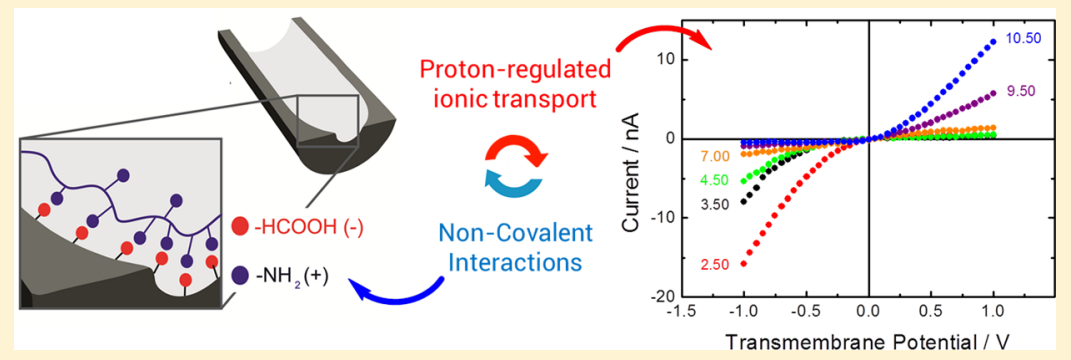

ABSTRACT: In this paper, the fabrication of a biomimetic nanofluidic diode whose ionic transport characteristics can be completely modulated with the proton concentration in solution is demonstrated. The fabrication procedure involves the electrostatic assembly of poly(allylamine hydrochloride) (PAH) into a track-etched conical nanochannel. A fully reversible, zwitterionic-like behavior with important implications for the supramolecular interactions of the PAH within confined spaces was observed. The experimental design constitutes a facile venue for the fabrication of functional nanofluidic devices and paves the way for a number of applications in nanofluidics and biosensing. Furthermore, in order to explain the experimental results and to obtain physicochemical information about the system, theoretical modeling using a continuous model based on PoissonNernst-Planck equations and a stochastic model using Monte Carlo simulations were performed. Good agreement between experiments and theory was found.

\section{INTRODUCTION}

Nanofluidics is an important emerging field within nanosciences that promises interesting applications in materials science, engineering, and biomedical research among other disciplines. ${ }^{1-4,4}$ The unique transport properties of solid-state nanocannels, which resemble several functional features of biological channels, such as ion selectivity and ionic-gating, has sparked the interest of "nanoscientists" worldwide and has increased their willingness to use these nanoarchitectures as nanofluidic elements. ${ }^{5-7}$

Several approaches to the construction of artificial nanometric fluidic devices have been developed, from biological to fully abiotic designs, ${ }^{8}$ each of them with particular advantages and disadvantages. Among the most common problems is the lack of mechanical stability exhibited by biological pores, and, in the case of artificial pores, the main issue is that the type of material that can be used strongly depends on the fabrication technique. For example, the focused ion beam technique demands that the thickness of the substrate that would contain the nanopore does not exceed few hundreds of nanometers.
In this regard, the track-etching method emerges as one of the most interesting procedures for obtaining abiotic nanochannels. The main advantage of this technique is its flexibility to tailor the geometry and size of the nanochannels. Furthermore, this technique can be adapted to a wide variety of materials. The procedure consists, first, of the irradiation of a dielectric foil with swift heavy ions. The ion tracks created by the ions are subsequently dissolved by chemical etching. Appropriate etching conditions are chosen depending on the template material. Thus, for example, for typical condensation polymers such as polycarbonate or polyethylene terephthalate, the etching procedure consists of an alkaline hydrolysis using highly concentrated alkali solutions. Ultimately, this procedure allows the reproducible fabrication of nanoscale channels, ${ }^{10}$ with tailored geometry (e.g., conical, cylindrical, biconical) and controlled diameter $(>10 \mathrm{~nm})$.

Received: February 20, 2017

Revised: April 3, 2017

Published: April 5, 2017 
The possibility of regulating the nanochannel geometry is of paramount importance because a longitudinal asymmetry in the geometry of a nanochannel yields an asymmetric ionic conductance that produces a diode-like, non-ohmic behavior, i.e., rectification in the ionic current. ${ }^{11,12}$ In addition, it has been shown that the rectification of ionic currents through nanochannels stems from changes in the selective transport of ions and, consequently, has a strong dependence on the surface charge of the channels, both in sign and magnitude. Therefore, in order to regulate the rectification of a nanochannel and the selective transport of ionic species it is necessary to regulate the surface charge of the nanochannel in a controlled manner. This can be done by a number of means, like electroless deposition or chemical functionalization as well as by sputtering and atomic layer deposition. ${ }^{13-17}$ This is the reason why surface modification of nanopores and nanochannels has become an important object of study of a number of research groups.

On the other hand, electrostatic self-assembly of polyelectrolytes represents a method that has been used in the past few years to obtain smart and functional devices in a variety of confined geometries. ${ }^{18}$ Even though promising applications of this supramolecular method have been shown (regarding its versatility and simplicity), the behavior of polyelectrolytes in confinement is not yet fully understood. ${ }^{19-21}$

Until now, the conformation of polyelectrolytes within nanochannels has been studied by means of gas-flow porometry and scanning electron microscopy. These investigations revealed that polyelectrolytes tend to form either nanowires or nanotubes for smaller and larger nanochannels diameters, respectively. ${ }^{22,23}$ However, these experiments were performed in dry conditions, and consequently, the information about the conformation of the polymers cannot be extrapolated to nanofluidic experiments in which polymers are in a hydrated state. In this regard, theoretical modeling and fitting can be used to obtain information about the effect of the confinement of the polymers in aqueous environments. There are several examples in the literature about the advantages of theoretical approaches to understand the behavior of species under nanometric confinement. ${ }^{24,25}$

In the present work, we have modified the surface of a solidstate nanochannel with the polycation poly(allylamine hydrochloride) (PAH) and studied the concomitant changes in the nanopore surface charge as a function of the concentration of protons in solution. As a result, a $\mathrm{pH}$-responsive nanofluidic device displaying a fully tunable and reversible ionic rectification was obtained and characterized. With regard to obtaining a complete physicochemical understanding of the system, experiments were accompanied by theoretical calculations based on the Poisson-Nernst-Planck (PNP) equations and Monte Carlo simulations (MC). To our knowledge, this is the first time that $\mathrm{MC}$ simulations have been applied to explain experimental results in nanofluidic systems modified with protonable species.

\section{EXPERIMENTAL METHODS}

Materials. Poly(allylamine hydrochloride) (PAH, $M_{\mathrm{w}}=15$ $\mathrm{kDa}$ ) was purchased from Sigma-Aldrich and used as received. Single-ion-irradiated PET foils (Hostaphan RN 12, Hoechst) of $12 \mu \mathrm{m}$ were irradiated at the linear accelerator UNILAC in GSI (Darmstadt, Germany, http://www.gsi.de) with swift heavy ions $\mathrm{Au}$ ) having an energy of $11.4 \mathrm{MeV}$ per nucleon.
Chemical Etching. Two different etching procedures were utilized to obtain single asymmetric nanochannels from iontracked foils. On the one hand, conical geometries using $9 \mathrm{M}$ sodium hydroxide as the etching solution and a mixture of $1 \mathrm{M}$ formic acid and $1 \mathrm{M} \mathrm{KCl}$ as stopping solution while applying an electro-stopping field of $1 \mathrm{~V}$ were obtained. The applied potential has two purposes: (a) it increases the nanochannel's cone angle and (b) it allows the monitoring of the pore opening by observing the current flowing through the nanochannels. ${ }^{26}$ On the other hand, to obtain more tapered geometries, a surfactant-assisted chemical etching was used. ${ }^{27}$ The foils were first exposed to UV light for $35 \mathrm{~h}$ on one side only. The foil is subsequently inserted in an electrochemical cell, separating two cell compartments. The compartment facing the UV-pre-exposed polymer side was filled with etching solution $(\mathrm{NaOH} 6 \mathrm{M})$, while the other side was filled with the etching solution $(\mathrm{NaOH} 6 \mathrm{M})$ containing a surfactant (DOWFAX 2A1, 0.04\%). The etching process was carried out at $60{ }^{\circ} \mathrm{C}$ for $6.5 \mathrm{~min}$.

Many single-track nanochannels fabricated following the first etching procedure had to be discarded because they did not show ionic conductance. They were either "closed" or they closed shortly after measurements started and reopened only after higher transmembrane voltages were applied. Moreover, the ion current rectification factor of these nanochannels was much smaller than those obtained by the surfactant-assisted procedure. The surfactant-assisted procedure resulted in bulletlike (BL) nanochannels exhibiting high rectification factors. Therefore, all experimental results reported here were obtained using BL nanochannels.

Modification with Poly(allylamine hydrochloride). Single nanochannel membranes were modified by dip coating in an aqueous $10 \mathrm{mM}$ poly(allylamine hydrochloride) solution (in monomer units) at a $\mathrm{pH} 6$ for $2 \mathrm{~h}$. The membranes were rinsed several times with distilled water and dried under ambient conditions.

Current-Voltage Measurements. Current-voltage curves were obtained using a potentiostat (Gamry Reference 600) with a four-electrode setup (working, working sense, reference and counter-electrode) to minimize the contribution from the processes occurring at the electrodes surfaces due to the voltage drop across the cell. ${ }^{28}$ In this way, we are able to measure conductance variations arising from changes in the nanochannel. Both the working and counter-electrode were made of platinum, while the reference and working sense were commercial silver/silver chloride electrodes. A homemade conductivity cell was fabricated to avoid leakage currents; i.e., the compartments are connected by a single nanochannel. In all of the experiments the working electrode was placed at the tip of the nanochannel while the counter-electrode was placed at the base. A $0.1 \mathrm{M} \mathrm{KCl}$ solution was used as electrolyte. The same experimental setup has been used in all the experiments in order to unambiguously correlate $I-V$ plots with nanopore surface charges.

Rectification Factor. A rectification factor $\left(f_{\text {rec }}\right)$, for quantifying the rectification efficiency, was defined. In all experiments, the definition for $f_{\text {rec }}$ was

$$
f_{\text {rec }}= \pm\left|\frac{I(1 V \text { or }-1 V)}{I(-1 V \text { or } 1 V)}\right|
$$

Here, the current in the numerator is the largest current value corresponding to the high conductance state, while the 

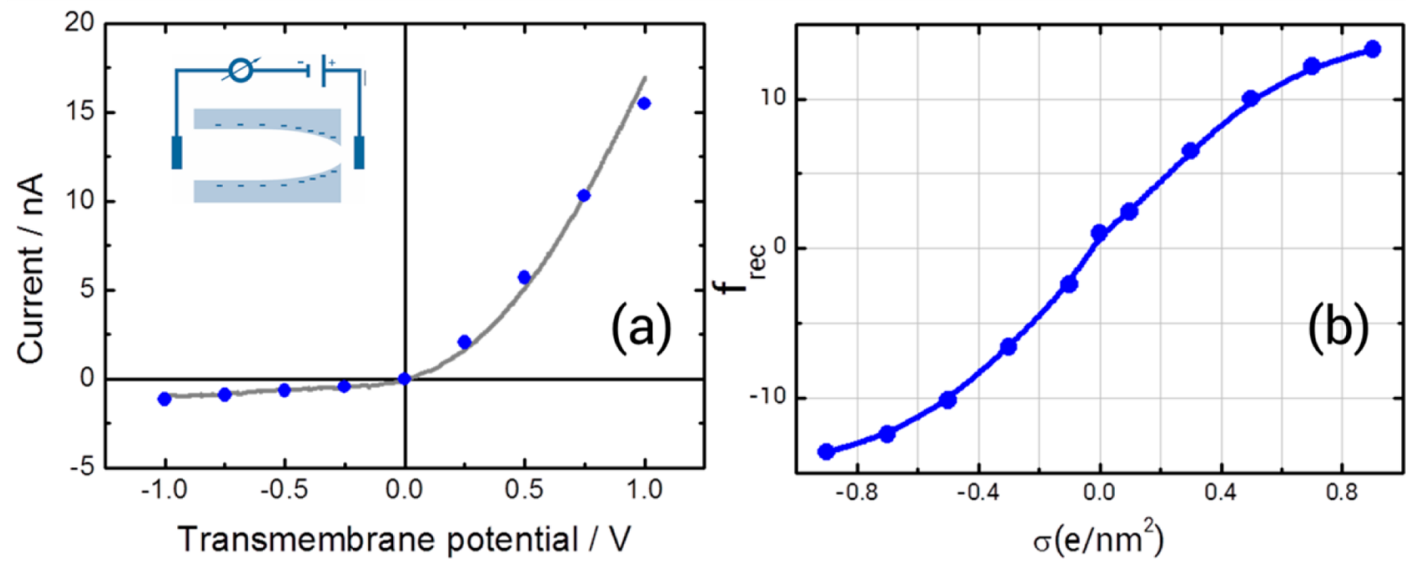

Figure 1. (a) Experimental results (gray line) and fitting with PNP model (blue dotted curve) for a bullet-like-etched PET nanochannel. Inset: Simplified scheme showing the electrode configuration. (b) Rectification factor versus surface charge density obtained from PNP theory for a bulletlike nanochannel. Tip diameter: $20 \mathrm{~nm}$. Base diameter: $500 \mathrm{~nm}$.

one in the denominator is the lowest current value corresponding to the low conductance state. Additionally, if the higher current corresponds to a positive voltage then the rectification factor is multiplied by -1 (Figure 1 ). This definition allows assigning a negative $f_{\text {rec }}$ for the case of negative surface charges and positive $f_{\text {rec }}$ for positive surface charges. This definition simplifies the interpretation of the experimental results in terms of surface charge.

Theoretical Methods. Analytical Model. In order to obtain information about the geometry and surface charge of the nanochannels after the modification with $\mathrm{PAH}$, a theoretical modeling based on the one-dimensional PNP formalism was used. $^{29}$ BL nanochannels were modeled using parameters obtained either experimentally or from literature. Large opening (base) diameters of $\sim 500 \mathrm{~nm}$ were obtained from scanning electron microscopy imaging of multipore membranes (Figure S1). Other parameters such as the longitudinal profile and the native surface charge of the nanochannel were obtained from the literature. The surface charge density of the nanochannel was found to be between 1.5 and $1.7 \mathrm{lel} / \mathrm{nm}^{2}$. With these parameters, the best fits to the experimental $I-V$ curves yielded a small opening (tip) diameter of $20 \mathrm{~nm}$.

The basic set of equations that were used to describe the transport through the nanochannels were the Nernst-Planck equation

$$
\vec{J}_{i}=-D_{i}\left(\nabla c_{i}+z_{i} c_{i} \nabla \phi\right)
$$

the Poisson equation

$$
\nabla^{2} \phi=-\frac{F^{2}}{\varepsilon R T} \sum_{i} z_{i} c_{i}
$$

and the continuity equation

$$
\nabla \vec{J}=0
$$

where $\vec{J}_{i}, D_{i}, z_{i}$ and $\varepsilon$ are the ionic flux, the diffusion coefficient, the ion charge, and the solution permittivity inside the channel, respectively.

Monte Carlo Simulations. MC simulations were performed in order to study the influence of the nanoconfinement on the acid-base equilibrium of a weak polyelectrolyte, i.e., $\mathrm{PAH}$. The acid-base equilibrium has a strong influence on the experimental output measured as transmembrane currents.
For these studies, a simulation box containing a $5 \mathrm{~nm}$ diameter pore with a length of $20 \mathrm{~nm}$ was used. Both the monomers of $\mathrm{PAH}$ and the native carboxylic acid groups in the walls of the nanochannel were considered as weak electrolytes with the following acid-base equilibriums

$$
\begin{aligned}
& -\left(\mathrm{NH}_{3}^{+}\right)_{n}-\leftrightharpoons-\left(\mathrm{NH}_{2}\right)_{n}-+n \mathrm{H}^{+} \\
& \mathrm{p} K_{\mathrm{a}}=10.4 \text { (monomers in the polymer chain) } \\
& \mathrm{R}-\mathrm{COOH} \leftrightharpoons \mathrm{R}-\mathrm{COO}^{-}+\mathrm{H}^{+} \\
& \mathrm{p} K_{a}=3.5 \text { (charges on the pore walls) }
\end{aligned}
$$

The acidic behavior was investigated in a grand canonical ensemble. To perform the simulations, a monomer was randomly chosen and an attempt was made to switch its charge state. The free energy change, $\Delta E$, governing the success of the attempt, can be described in terms of two major contributions: the change in the Coulombic interactions between monomers, cations and anions, $\Delta E_{\text {total }}$ and the free energy change corresponding to the acid-base reaction of an isolated monomer ${ }^{30,31}$

$$
\Delta E=\Delta E_{\text {total }} \pm k_{\mathrm{B}} T \log \left(\mathrm{pH}-\mathrm{p} K_{i}\right)
$$

where $\mathrm{pH}$ is the $\mathrm{pH}$ of the system and $\mathrm{p} K_{\mathrm{i}}$ is the intrinsic $\mathrm{p} K_{\mathrm{a}}$ of the monomer or the carboxylic groups on the pore walls. The plus sign in the equation is used when the monomer is protonated and the minus sign when it is deprotonated.

\section{RESULTS AND DISCUSSION}

Asymmetric nanochannels display the ability to rectify ionic current passing through them as a function of the sign and magnitude of the surface charges exposed on their walls. ${ }^{32}$ This occurs, in principle, because charged nanochannels are highly selective to ionic species of opposite sign of the surface. ${ }^{33}$ However, the mechanism underlying the rectification of ionic currents is a more complex phenomenon. We have recently shown that the transport number of cations in a conically etched PET nanochannel, that bears negative surface charges after etching, is close to 0.95 at negative voltages (low conductance state) but close to 0.5 at positive voltages (high conductance states) meaning that the emergence of rectification is due to a loss of the selectivity at positive potentials 


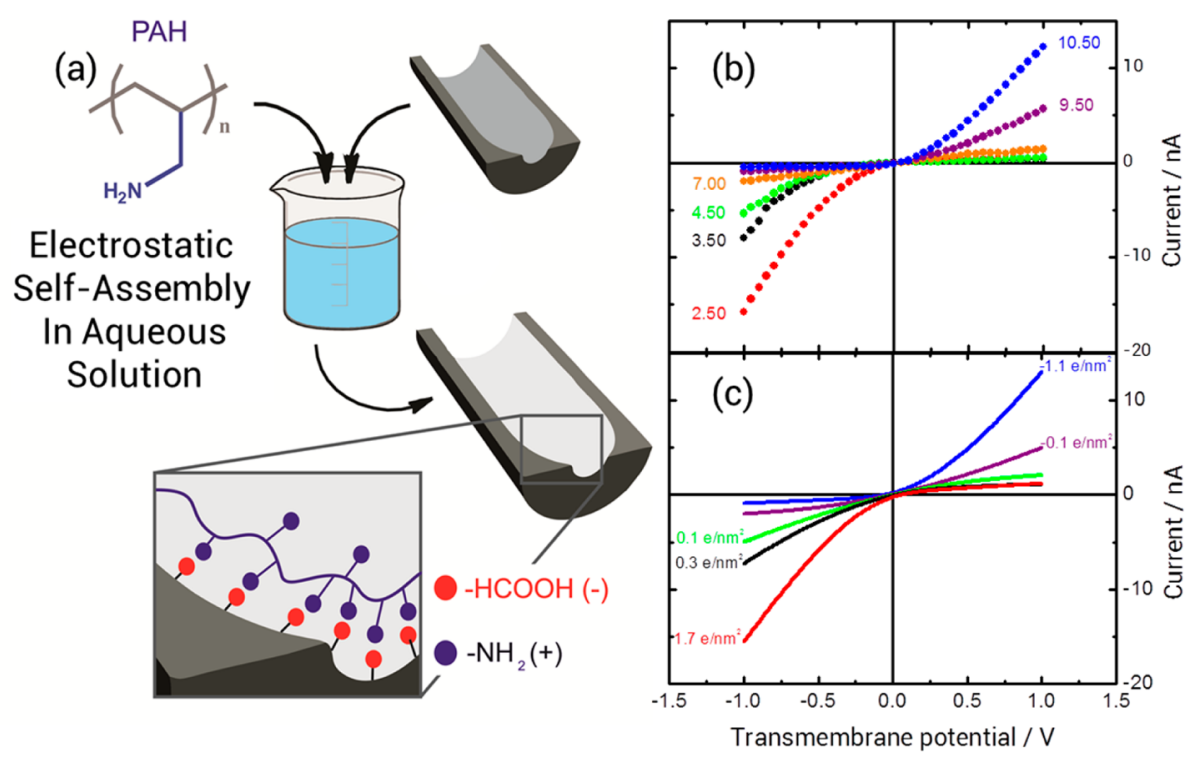

Figure 2. (a) Scheme showing the modification of the nanochannel with PAH and the ionizable groups in the surface. (b) Experimental curves of the modified nanochannel under different $\mathrm{pH}$ conditions $(\mathrm{c})$. Theoretical curves of the modified nanochannel at different surface charges.

allowing both anions and cations to contribute to the transport of current. ${ }^{34}$

The current-voltage measurements of the nanochannel without any further modification correspond to a negatively charged surface (Figure 1a). Once the PNP modeling was performed, an excellent agreement between experimental and theoretical results was observed (Figure 1a). The PNP theory correlates the current-voltage characteristics (i.e., the rectification efficiency) of a given nanochannel with the surface charge. For a fixed geometry, we observed a monotonic increase in the rectification factor upon increasing the surface charge (Figure 1b), which confirms that a functional molecule that can finely modify its state of charge can consequently transduce these changes into the direction and magnitude of the ionic transport.

The native negative charge of the nanochannels stems from the chemical nature of PET. The hydrolysis performed during the etching of the nanochannel leads to the appearance of carboxylate groups on the surface. These negative groups were used for the electrostatic assembly of $\mathrm{PAH}$, a polycation that strongly interacts with negative species (Figure 2).

After $\mathrm{PAH}$ modification, $I-V$ curves at different $\mathrm{pH}$ values were measured. Figure 2 shows the $I-V$ curves for a PAHmodified nanochannel in $0.1 \mathrm{M} \mathrm{KCl}$ measured under different conditions and the simulated curves using the PNP model. The change in the shape of the $I-V$ curves follows a continuous trend that can be clearly observed in the $f_{\text {rec }}$ vs $\mathrm{pH}$ plots (Figure 3 ). In the $\mathrm{pH}$ range of $4-8$ a reversion in the rectification direction, i.e., reversion of $f_{\text {rec }}$ sign was observed. This result was reproduced on different nanochannels.

The behavior of the rectification factor with $\mathrm{pH}$ showed an extended low rectification region between $\mathrm{pH} 5$ and 8 . The PNP model was then used to correlate rectification factors with the surface charge density on the nanochannel walls, finding that this region exhibits a net charge close to zero. For this reason, we chose to refer to this region as the isoelectric region (IR) (Figure 3). In order to quantify the variation in magnitude and sign of the surface charge with $\mathrm{pH}$, the charge was calculated for each experimental curve measured under different $\mathrm{pH}$ conditions (Figure $3 \mathrm{~b}$ ).
The maximum value obtained for a nanochannel modified with $\mathrm{PAH}$ at low $\mathrm{pH}$ was $1.7 \mathrm{lel} / \mathrm{nm}^{2}$, which is close to the native anionic charge density of the nanochannel. On the other hand, the maximum negative surface charge obtained after the modification at high $\mathrm{pH}$ was $-1.1 \mathrm{el} / \mathrm{nm}^{2}$, which is slightly lower than the initial surface charge of the nanochannel. This difference can be explained taking into account that negative charges can only arise from the PET surface underneath the $\mathrm{PAH}$ layer. After the modification, at high $\mathrm{pH}$, the $\mathrm{PAH}$ starts to deprotonate, until the net charge becomes negative due to the ionized carboxylate groups present on the PET foil. The fact that this net negative charge is lower than the initial value can be explained by considering that some groups of the PAH must remain protonated even at high $\mathrm{pH}$. This was indeed confirmed by MC simulations. They showed that the PAH remains slightly protonated (charged) even at very high $\mathrm{pH}$ values (Figure 3 ). Figure $3 \mathrm{c}$ shows $10 \%$ of the $\mathrm{PAH}$ amine groups is still protonated at $\mathrm{pH}$ values as high as 12 . Furthermore, considering the electrostatic charges stemming from the protonated polymer and the surface it was possible to calculate the contributions to the surface charge arising from the adsorbed polymer and the nanochannel surface (Figure $3 \mathrm{~b}$ ).

From these results, it can be seen that within the IR both the native nanochannel carboxylic groups and the adsorbed polycation remain in a highly charged state, strongly interacting with each other.

The isoelectric region was also studied using MC simulations after calculating the net charge inside the channel (Figure $3 \mathrm{~b}$, red curve). From MC simulations we concluded that the region between $\mathrm{pH} 5$ and 8 (IR) can be interpreted as a region where there is significant negative charge from carboxylate groups that are highly deprotonated (Figure 3c, green curve) and positive charges from the $\mathrm{PAH}$ amine groups which, in turn, are highly protonated (Figure 3c, blue curve). Even though both groups are strongly charged, local electrostatic neutralization occurs and the net charge is zero.

Previous works have theoretically and experimentally shown that the apparent $\mathrm{p} K_{\mathrm{a}}$ of a weak polyelectrolyte shifts by increasing the confinement. For the case of polybases like the $\mathrm{PAH}$ a shift in the $\mathrm{p} K_{\mathrm{a}}$ toward higher values is expected due to 

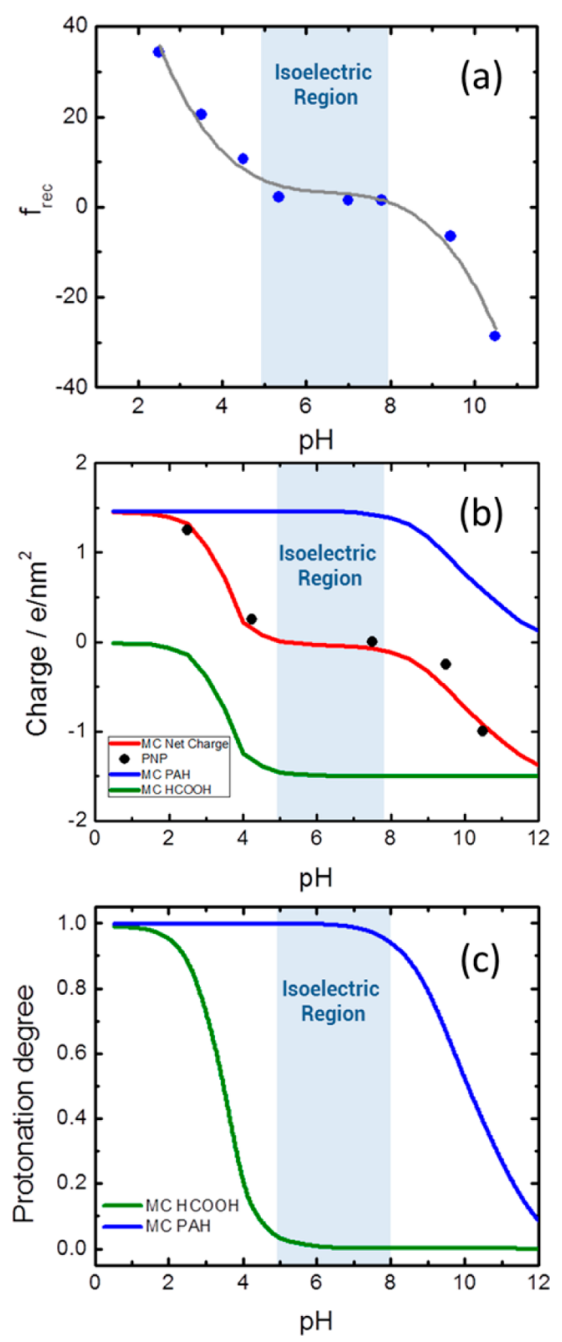

Figure 3. (a) Rectification factor as a function of $\mathrm{pH}$ calculated using eq 1. (b) Surface charge density versus $\mathrm{pH}$ as derived from (i) the fitting of the experimental $I-V$ curves using the PNP formalism, (ii) MC simulations, and (iii) the fitting between experimental and PNP $I-V$ curves. (c) Protonation degree vs $\mathrm{pH}$ obtained from $\mathrm{MC}$ simulations.

loss of degrees of freedom. ${ }^{35-38}$ These previous observations are in agreement with our results. It is important to note that both PNP and MC methods are in excellent agreement with each other and also with the experimental results.

Even though the PNP model allowed us to accurately fit experimental results, it evidenced difficulties at extreme $\mathrm{pH}$ values. The maximum rectification factor obtained for the simulations was 15 while the experimental maximum values were $\sim 30$. The difference between experimental and theoretical values stems mainly from ionic currents in the lower conductivity state. For the calculation of rectification factors, small differences in these current values may produce large variations in the $f_{\text {rec }}$ values (eq 1). Similar differences have been reported previously. ${ }^{39,27,29}$ One of the causes of these discrepancies could be an inhomogeneity on the surface charge that might exist in real nanochannels and that is not taken into account in the theory.

Also, it is important to take into account that the PNP model assumes that the charge is one-dimensional while in our experiments the charge is expected to have a tridimensional distribution due to the presence of the adsorbed polymer. ${ }^{40}$ In this regard, it was theoretically shown that in nanochannels modified with polyelectrolytes with a degree of polymerization of 40 rectification factors up to 42 can be reached. ${ }^{39}$ Further research needs to be performed in order to address these questions.

Another important issue to address was the system's reversibility, i.e., the capacity of exposing the system to successive cycles of extreme $\mathrm{pH}$ values without losing its responsiveness. It has been shown that in planar substrates, the application of extreme $\mathrm{pH}$ and ionic strength conditions can lead to the disassembly of polyelectrolyte multilayers, especially when constituted of weak polyelectrolytes. ${ }^{41-43}$ This feature has been used for drug delivery by controlling the disassembly of multilayered structures containing specific molecules inside. However, in the case of nanofluidic applications $\mathrm{pH}$-induced disassembly of the functional layer is undesired. Testing the reversibility by changing the $\mathrm{pH}$ of the electrolyte solution successively between 2.5 and 10.5 confirmed the robustness of the modification procedure (Figure 4).

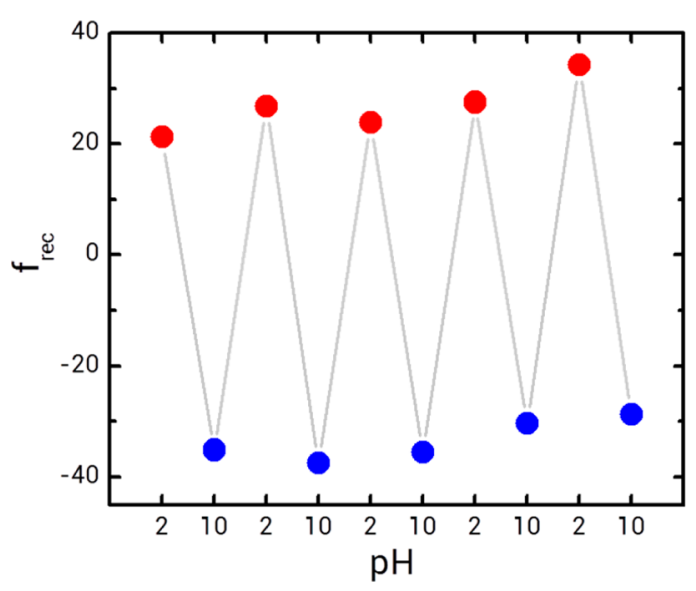

Figure 4. Reversibility test: The frec were calculated from experiments measured at extreme $\mathrm{pH}$ successively. A high reversibility is observed.

High rectification efficiencies were obtained for each cycle. Normal $f_{\text {rec }}$ for surface modified nanochannels are around $\sim 5$. We obtained $f_{\text {rec }}$ of $\sim 30$ which are comparable with the ones obtained for nanochannels modified with densely charged polymer brushes that required more complex synthetic protocols. The fact that the $\mathrm{PAH}$ remains inside the nanochannel even upon the exposure to extreme $\mathrm{pH}$ can be explained considering the protonation degree of the polyelectrolyte obtained from MC simulations (Figure 3). These results reveal that at low $\mathrm{pH} \mathrm{PAH}$ is fully protonated, while the carboxylate groups are not. On the other hand, at high $\mathrm{pH}$, the carboxylate groups are fully deprotonated while the $\mathrm{PAH}$ is not. However, even at extreme $\mathrm{pH}$ values such as 2 or 10 there are carboxylate and amine groups that still remain unprotonated or protonated, respectively, allowing electrostatic interactions to restrain the PAH from leaving the channel.

The functional features of this experimental system closely resemble those observed in biological KcsA ion channels. This family of ionic channel proteins shows $\mathrm{pH}$ and voltagecontrolled ion gating. ${ }^{44}$ As can be seen in Figure 5, there is a $\mathrm{pH}$-induced on-off switch at a fixed voltage. This behavior is then reversed by applying the opposite voltage. This means that by applying different combinations of $\mathrm{pH}$ and voltage the selective transport of ionic species can be fully regulated. 


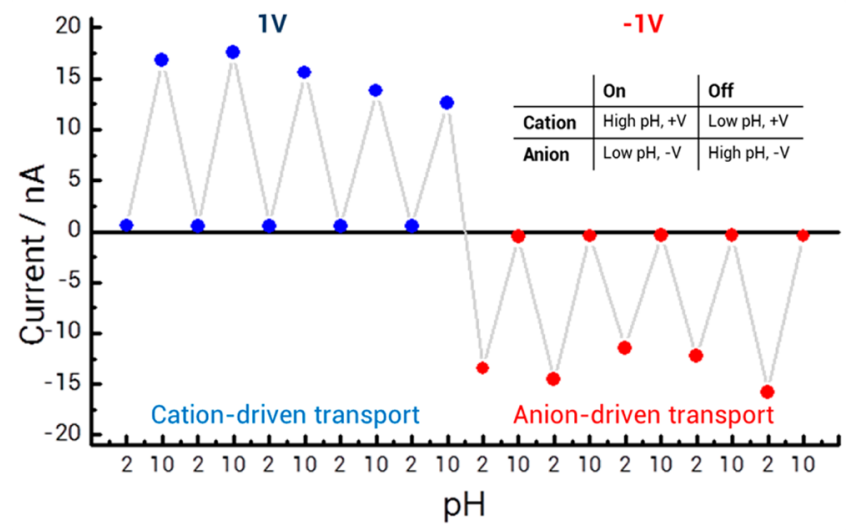

Figure 5. Ionic currents measured at different $\mathrm{pH}$ and transmembrane voltages. These results show the gating capacity of a PAH-modified nanochannel. Different combinations of $\mathrm{pH}$ and voltage allow control of the selective passage of ions through the nanochannel.

\section{CONCLUSIONS}

We demonstrated a simple and highly effective way to control the ionic transport properties of asymmetric nanochannels in the presence of different proton concentrations in solution. The procedure consisted of coating a hydrolyzed nanochannelcontaining foil with a weak polycation, poly(allylamine hydrochloride). Poisson-Nernst-Planck equations were used to calculate the surface charge densities under different $\mathrm{pH}$ conditions, and Monte Carlo simulations were performed to study the relationship between the protonation degrees of the $\mathrm{PAH}$ layer and the ionizable surface groups on the nanochannel wall, and the solution $\mathrm{pH}$. We showed that the ionic transport properties of the nanochannel are governed by a delicate interplay between the negatively charged carboxylic groups of the nanochannel walls and the positively charged primary amines on the PAH layer. It is shown that the shift in the protonation degree of amino and carboxyl groups prevents the removal of the PAH layer from the nanochannel due to presence of residual charges, even in the case of extreme $\mathrm{pH}$ conditions. Furthermore, the capabilities of the system to act as a biomimetic $\mathrm{pH}$ - and voltage-controlled ionic gate were demonstrated. Taking into account that simple derivatization of pendant amino groups of the polyallylamine layer with different chemical groups can endow nanochannels with predefined responsiveness or multiple functions, we believe that these results can inspire further research toward advanced design of nanofluidic devices.

\section{ASSOCIATED CONTENT}

\section{S Supporting Information}

The Supporting Information is available free of charge on the ACS Publications website at DOI: 10.1021/acs.jpcc.7b01639.

SEM images of multitrack foils used for performing the PNP calculations (PDF)

\section{AUTHOR INFORMATION}

\section{Corresponding Authors}

*E-mail: gperezmitta@inifta.unlp.edu.ar. Web: https:// softmatter.quimica.unlp.edu.ar and https://www.facebook. com/SoftMatterLaboratory/.
*E-mail: azzaroni@inifta.unlp.edu.ar. Web: https://softmatter. quimica.unlp.edu.ar and https://www.facebook.com/ SoftMatterLaboratory/.

ORCID

Omar Azzaroni: 0000-0002-5098-0612

Notes

The authors declare no competing financial interest.

\section{ACKNOWLEDGMENTS}

We acknowledge financial support from ANPCyT (PICT 20102554, PICT-2013-0905 and PPL 2011-003) and from the Deutsche Forschungsgemeinschaft (DFG-FOR 1583). M.E.T.$M$ and C.T. acknowledge support by the LOEWE project iNAPO funded by the Hessen State Ministry of Higher Education, Research and Arts. G.P.-M. and F.M.G. acknowledge CONICET for a doctoral fellowship. A.A. and O.A. are staff members of CONICET.

\section{REFERENCES}

(1) Hou, X.; Zhang, H.; Jiang, L. Building Bio-Inspired Artificial Functional Nanochannels: From Symmetric to Asymmetric Modification. Angew. Chem., Int. Ed. 2012, 51, 5296-5307.

(2) Venkatesan, B. M.; Bashir, R. Nanopore Sensors for Nucleic Acid Analysis. Nat. Nanotechnol. 2011, 6, 615-624.

(3) Hou, X. Smart Gating Multi-Scale Pore/Channel-Based Membranes. Adv. Mater. 2016, 28, 7049-7064.

(4) Kong, Y.; Fan, X.; Zhang, M. H.; Hou, X.; Liu, Z. Y.; Zhai, J.; Jiang, L. Nanofluidic Diode Based on Branched Alumina Nanochannels with Tunable Ionic Rectification. ACS Appl. Mater. Interfaces 2013, 5, 7931-7936.

(5) Siwy, Z.; Apel, P. Y.; Baur, D.; Dobrev, D. D.; Korchev, Y. E.; Neumann, R.; Spohr, R.; Trautmann, C.; Voss, K.-O. Preparation of Synthetic Nanopores with Transport Properties Analogous to Biological Channels. Surf. Sci. 2003, 532, 1061-1066.

(6) Yameen, B.; Ali, M.; Neumann, R.; Ensinger, W.; Knoll, W.; Azzaroni, O. Ionic Transport Through Single Solid-State Nanopores Controlled with Thermally Nanoactuated Macromolecular Gates. Small 2009, 5, 1287-1291.

(7) Zhang, H.; Hou, X.; Yang, Z.; Yan, D.; Li, L.; Tian, Y.; Wang, H.; Jiang, L. Bio-inspired Smart Single Asymmetric Hourglass Nanochannels for Continuous Shape and Ion Transport Control. Small 2015, 11, 786-791.

(8) Hou, X.; Guo, W.; Jiang, L. Biomimetic Smart Nanopores and Nanochannels. Chem. Soc. Rev. 2011, 40, 2385-2401.

(9) Kocer, A.; Tauk, L.; Dejardin, P. Nanopore sensors: From Hybrid to Abiotic Systems. Biosens. Bioelectron. 2012, 38, 1-10.

(10) Fleischer, R. L.; Price, P. B.; Walker, R. M. Nuclear Tracks in Solids: Principles and Applications; University of California Press: Berkeley, 1975.

(11) Siwy, Z.; Apel, P. Y.; Dobrev, D.; Neumann, R.; Spohr, R.; Trautmann, C.; Voss, K.-O. Ion Transport Through Asymmetric Nanopores Prepared by Ion Track Etching. Nucl. Instrum. Methods Phys. Res., Sect. B 2003, 208, 143-148.

(12) Siwy, Z.; Dobrev, D.; Neumann, R.; Trautmann, C.; Voss, K.-O. Electro-Responsive Asymmetric Nanopores in Polyimide with Stable Ion-Current Signal. Appl. Phys. A: Mater. Sci. Process. 2003, 76, 781785.

(13) Martin, C. R. Membrane-Based Synthesis of Nanomaterials. Chem. Mater. 1996, 8, 1739-1746.

(14) Yameen, B.; Ali, M.; Neumann, R.; Ensinger, W.; Knoll, W.; Azzaroni, O. Synthetic Proton-Gated Ion Channels via Single SolidState Nanochannels Modified with Responsive Polymer Brushes. Nano Lett. 2009, 9, 2788-2793.

(15) Zhang, H.; Hou, X.; Hou, J.; Zeng, L.; Tian, Y.; Li, L.; Jiang, L. Synthetic Asymmetric-Shaped Nanodevices with Symmetric pHGating Characteristics. Adv. Funct. Mater. 2015, 25, 1102-1110. 
(16) Hou, X.; Liu, Y.; Dong, H.; Yang, F.; Li, L.; Jiang, L. A pHGating Ionic Transport Nanodevice: Asymmetric Chemical Modification of Single Nanochannels. Adv. Mater. 2010, 22, 2440-2443.

(17) Spende, A.; Sobel, N.; Lukas, M.; Zierold, R.; Riedl, J. C.; Gura, L.; Schubert, I.; Montero Moreno, J. M.; Nielsch, K.; Stühn, B. TiO2, $\mathrm{SiO} 2$, and $\mathrm{Al} 2 \mathrm{O} 3$ Coated Nanopores and Nanotubes Produced by ALD in Etched Ion-Track Membranes for Transport Measurements. Nanotechnology 2015, 26, 335301-335312.

(18) Azzaroni, O.; Lau, K. H. A. Layer-by-Layer Assemblies in Nanoporous Templates: Nano-Organized Design and Applications of Soft Nanotechnology. Soft Matter 2011, 7, 8709-8724.

(19) Lee, D.; Nolte, A. J.; Kunz, A. L.; Rubner, M. J.; Cohen, R. E. PH-Induced Hysteretic Gating of Track-Etched Polycarbonate Membranes: Swelling/Deswelling Behavior of Polyelectrolyte Multilayers in Confined Geometry. J. Am. Chem. Soc. 2006, 128, 85218529.

(20) Ali, M.; Yameen, B.; Neumann, R.; Ensinger, W.; Knoll, W.; Azzaroni, O. Biosensing and Supramolecular Bioconjugation in Single Conical Polymer Nanochannels. Facile Incorporation of Biorecognition Elements into Nanoconfined Geometries. J. Am. Chem. Soc. 2008, 130, 16351-16357.

(21) Ali, M.; Yameen, B.; Cervera, J.; Ramírez, P.; Neumann, R.; Ensinger, W.; Knoll, W.; Azzaroni, O. Layer-by-Layer Assembly of Polyelectrolytes into Ionic Current Rectifying Solid-State Nanopores: Insights from Theory and Experiment. J. Am. Chem. Soc. 2010, 132, $8338-8348$.

(22) Roy, C. J.; Dupont-Gillain, C.; Demoustier-Champagne, C.; Jonas, A. M.; Landoulsi, J. Growth Mechanism of Confined Polyelectrolyte Multilayers in Nanoporous Templates. Langmuir 2010, 26, 3350-3355.

(23) Alem, H.; Blondeau, F.; Glinel, K.; Demoustier-Champagne, S.; Jonas, A. M. Layer-by-Layer Assembly of Polyelectrolytes in Nanopores. Macromolecules 2007, 40, 3366-3372.

(24) Cervera, J.; Schiedt, B.; Ramırez, P. A. Poisson/Nernst-Planck Model for Ionic Transport Through Synthetic Conical Nanopores. Europhys. Lett. 2005, 71, 35-41.

(25) Sparreboom, W.; Van den Berg, A.; Eijkel, J. C. T. Transport in Nanofluidic Systems: A Review of Theory and Applications. New J. Phys. 2010, 12, 015004-015027.

(26) Siwy, Z.; Fulinski, A. A Nanodevice for Rectification and Pumping ions. Am. J. Phys. 2004, 72, 567-574.

(27) Apel, P. Y.; Blonskaya, I. V.; Levkovich, N. V.; Orelovich, O. L. Asymmetric Track Membranes: Relationship Between Nanopore Geometry and Ionic Conductivity. Pet. Chem. 2011, 51, 555-567.

(28) Application note: http://www.gamry.com/application-notes/ electrodes-cells/two-three-and-four-electrode-experiments/.

(29) Cervera, J.; Schiedt, B.; Neumann, R.; Mafé, S.; Ramírez, P. Ionic, Ionic Conduction, Rectification, and Selectivity in Single Conical Nanopores. J. Chem. Phys. 2006, 124, 104706.

(30) Albesa, A. G.; Rafti, M.; Vicente, J. L. Trivalent Cations Switch the Selectivity in Nanopores. J. Mol. Model. 2013, 19, 2183-2188.

(31) Barr, S. A.; Panagiotopoulos, A. Z. Conformational Transitions of Weak Polyacids Grafted to Nanoparticles. J. Chem. Phys. 2012, 137, 144704.

(32) Harrell, C. C.; Siwy, Z. S.; Martin, C. R. Conical Nanopore Membranes: Controlling the Nanopore Shape. Small 2006, 2, 194198.

(33) Siwy, Z. S.; Martin, C. R. Tuning Ion Current Rectification in Synthetic Nanotubes. Lect. Notes Phys. 2007, 711, 349-365.

(34) Pérez-Mitta, G.; Albesa, A. G.; Toimil-Molares, M. E.; Trautmann, C.; Azzaroni, O. The Influence of Divalent Anions on the Rectification Properties of Nanofluidic Diodes: Insights from Experiments and Theoretical Simulations. ChemPhysChem 2016, 17, $2718-2725$.

(35) Longo, G. S.; Olvera de La Cruz, M.; Szleifer, I. Molecular Theory of Weak Polyelectrolyte Gels: The Role of $\mathrm{pH}$ and Salt Concentration. Macromolecules 2011, 44, 147-158.
(36) Choi, J.; Rubner, M. F. Influence of The Degree of Ionization on Weak Polyelectrolyte Multilayer Assembly. Macromolecules 2005, 38, 116-124.

(37) Itano, K.; Choi, J.; Rubner, M. F. Mechanism of the pH-Induced Discontinuous Swelling/Deswelling Transitions of Poly(allylamine hydrochloride)-Containing Polyelectrolyte Multilayer Films. Macromolecules 2005, 38, 3450-3460.

(38) Petrov, A. I.; Antipov, A. A.; Sukhorukov, G. B. Base-Acid Equilibria in Polyelectrolyte Systems: From Weak Polyelectrolytes to Interpolyelectrolyte Complexes and Multilayered Polyelectrolyte Shells. Macromolecules 2003, 36, 10079-10086.

(39) Tagliazucchi, M.; Rabin, Y.; Szleifer, I. Transport Rectification in Nanopores with Outer Membranes Modified with Surface Charges and Polyelectrolytes. ACS Nano 2013, 7, 9085-9097.

(40) Tagliazucchi, M.; Azzaroni, O.; Szleifer, I. Responsive Polymers End-Tethered in Solid-State Nanochannels: When Nanoconfinement Really Matters. J. Am. Chem. Soc. 2010, 132, 12404-12411.

(41) Lynn, D. M. Peeling Back the Layers: Controlled Erosion and Triggered Disassembly of Multilayered Polyelectrolyte Thin Films. Adv. Mater. 2007, 19, 4118-4130.

(42) Sukhishvili, S. A.; Granick, S. Layered, Erasable, Ultrathin Polymer Films. J. Am. Chem. Soc. 2000, 122, 9550-9551.

(43) Dubas, S. T.; Farhat, T. R.; Schlenoff, J. B. Multiple Membranes from "True" Polyelectrolyte Multilayers. J. Am. Chem. Soc. 2001, 123, $5368-5369$

(44) Hirano, M.; Onishi, Y.; Yanagida, T.; Ide, T. Role of the KcsA Channel Cytoplasmic Domain in pH-Dependent Gating. Biophys. J. 2011, 101, 2157-2162. 